\title{
Suppressive effects of a proton beam on tumor growth and lung metastasis through the inhibition of metastatic gene expression in 4T1 orthotopic breast cancer model
}

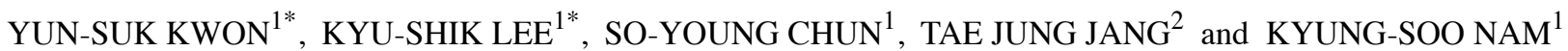 \\ ${ }^{1}$ Intractable Disease Research Center and Department of Pharmacology and ${ }^{2}$ Pathology, \\ School of Medicine, Dongguk University, 38066 Gyeongju, Republic of Korea
}

Received March 14, 2016; Accepted April 25, 2016

DOI: $10.3892 /$ ijo.2016.3520

\begin{abstract}
A proton beam is a next generation tool to treat intractable cancer. Although the therapeutic effects of a proton beam are well known, the effect on tumor metastasis is not fully described. Here, we investigated the effects of a proton beam on metastasis in highly invasive 4T1 murine breast cancer cells and their orthotopic breast cancer model. Cells were irradiated with $2,4,8$ or 16 Gy proton beam, and changes in cell proliferation, survival, and migration were observed by MTT, colony forming and wound healing assays. 4T1 breast cancer cell-implanted BALB/c mice were established and the animals were randomly divided into 4 groups when tumor size reached $200 \mathrm{~mm}^{3}$. Breast tumors were selectively irradiated with 10,20 or $30 \mathrm{~Gy}$ proton beam. Breast tumor sizes were measured twice a week, and breast tumor and lung tissues were pathologically observed. Metastasis-regulating gene expression was assessed with quantitative RT-PCR. A proton beam dose-dependently decreased cell proliferation, survival and migration in 4T1 murine breast cancer cells. Also, growth of breast tumors in the 4T1 orthotopic breast cancer model was significantly suppressed by proton beam irradiation without significant change of body weight. Furthermore, fewer tumor nodules metastasized from breast tumor into lung in mice irradiated with $30 \mathrm{~Gy}$ proton beam, but not with 10 and $20 \mathrm{~Gy}$, than in control. We observed correspondingly lower expression levels of urokinase plasminogen activator (uPA), uPA receptor, cyclooxygenase (COX)-2, and vascular endothelial growth factor (VEGF), which are important factors in cancer metastasis, in breast tumor irradiated with $30 \mathrm{~Gy}$ proton beam.
\end{abstract}

Correspondence to: Professor Kyung-Soo Nam, Department of Pharmacology and Intractable Disease Research Center, School of Medicine, Dongguk University, 38066 Gyeongju, Republic of Korea E-mail:namks@dongguk.ac.kr

"Co-first authors

Key words: proton beam, metastasis, breast cancer, metastatic gene, $4 \mathrm{~T} 1$ orthotopic breast cancer model
Proton beam irradiation did not affect expressions of matrix metalloproteinase (MMP)-9 and MMP-2. Taken together, the data suggest that, although proton beam therapy is an effective tool for breast cancer treatment, a suitable dose is necessary to prevent metastasis-linked relapse and poor prognosis.

\section{Introduction}

Although the 5-year survival rate of breast cancer patients is increasing due to advanced therapeutic techniques, lower survival rate and poor prognosis are still observed in patients diagnosed with metastatic breast cancer (1). Actually, 98\% of breast cancer patients with a localized tumor can survive 5 years, but just $27 \%$ of patients diagnosed with metastatic breast cancer can live $>5$ years (2). This indicates the importance of cancer metastasis prevention to improve therapeutic efficacy in breast cancer patients.

To metastasize, cancer cells must be released from the primary tumor and travel to a distant site by attachment and invasion through degradation of biological barriers, such as basement membrane (BM) and extracellular matrix (ECM). The degradation is predominantly regulated by membranedegrading enzymes, such as matrix metalloproteinases (MMPs), urokinase plasminogen activator (uPA), and uPA receptor (UPAR) (3). Among MMPs, the gelatinases MMP-2 and -9 play important roles in breast cancer metastasis and angiogenesis (4). In breast cancer cell systems, several studies have revealed that metastatic potential was suppressed by inhibition of MMP-2 and/or -9 (4-6). Also, a clinical study showed that expression levels of MMP-2 and -9 in metastatic breast cancer patients were higher than those in non-metastatic breast cancer patients (7). uPA is also closely related to metastatic potential. uPA accelerates ECM degradation and MMP-9 activation through the catalysis of plasmin production from plasminogen and it is also a well-known prognostic factor in breast cancer $(8,9)$. In clinical evaluation, poor prognosis and high rate of relapse were shown in node-negative breast cancer patients with high levels of uPA in breast tumor tissue or high concentrations of serum uPA, whereas patients with low levels of tissue uPA had a low risk of recurrence $(8,10,11)$. Consequently, the inhibition of these proteases is a key strategy to prevent breast cancer metastasis. 
Proton beam therapy is a promising radiotherapeutic tool due to the characteristic delivery of specifically designed doses to tumor tissue with minimized damage to surrounding normal tissues because the ionizing proton beam is deposited in an exact region with a dramatic end point increase of the specific energy per unit in a body (Bragg Peak) (12). Proton beam therapy is widely applied to treat various types of cancer including breast cancer (13). In general, the molecular biological study of therapeutic effects in a proton beam has focused on tumor cell apoptosis caused by direct or indirect DNA damage $(14,15)$. However, the effects on metastasis, a major cause of recurrence and poor prognosis, have not been fully investigated.

We previously showed that a proton beam diminished the metastatic potential of MCF-7 and MDA-MB-231 human breast cancer cells via the inhibition of MMP-9 activity and expression $(12,16)$. Furthermore, proton beam irradiation suppressed angiogenic activity in MCF-7 human breast cancer cells through the inhibition of vascular endothelial growth factor (VEGF) expression, an important angiogenic factor (17). Here, we investigated the efficacy of proton beam irradiation on breast cancer metastasis using aggressive 4T1 murine breast cancer cells isolated from naturally developed mammary tumor in BALB/c mice and its orthotopic breast cancer model $(18,19)$. We analyzed the effects of proton beam irradiation on cell proliferation, viability and metastatic potential in 4T1 murine breast cancer cells and also measured tumor growth, lung metastasis and metastatic gene mRNA expression in 4T1 orthotopic animal models.

\section{Materials and methods}

Cell culture. The 4T1 murine breast cancer cell line was obtained from the American Type Culture Collection (Rockville, MD, USA) and routinely maintained in Dulbecco's modified Eagle's medium (DMEM, Welgene, Daegu, Korea) supplemented with $10 \%$ fetal bovine serum (FBS) (Invitrogen, Carlsbad, CA, USA) and $1 \%$ antibiotic-antimycotic solution (Welgene) in $5 \%(\mathrm{v} / \mathrm{v}) \mathrm{CO}_{2}$ atmosphere at $37^{\circ} \mathrm{C}$.

Cell proliferation assay. To evaluate the effect of proton beam irradiation on cell proliferation, we conducted 3-(4,5-dimethylthiazol-2-yl)-2,5 diphenyl-tetrazolium bromide (MTT) assays. 4T1 murine breast cancer cells were plated at 2,000 cells per well into 96-well plates and incubated for $24 \mathrm{~h}$. Cells were then irradiated with a dose of 2, 4, 8, or 16 Gy by spread-out Bragg peak (SOBP) with the $100 \mathrm{MeV}$ proton accelerator (Korea Multi-Purpose Accelerator Complex, Gyeongju, Korea), grown for 24 or $48 \mathrm{~h}$, and then incubated with MTT for $4 \mathrm{~h}$ in the dark. Produced insoluble formazan was dissolved in dimethyl sulfoxide (DMSO) and the absorbance of formazan was measured at $570 \mathrm{~nm}$.

Clonogenic cell survival assay. Clonogenic cell viability was assessed by colony forming assay. Three hundred cells were seeded in each well of a 6-well plate and attached for $24 \mathrm{~h}$. Then, the cells were irradiated with a proton beam in a dose-dependent manner and additionally grown for 6 days The colonies were fixed with $10 \%$ formalin for $20 \mathrm{~min}$, stained with $1 \%$ crystal violet for $40 \mathrm{~min}$ and then captured.
Wound healing assay. Cell migration was evaluated with a wound healing assay. Cells were plated on 6-well plates coated with collagen IV (Corning, Bedford, MA, USA) and cultured to confluence. Cell monolayers were scratched with a blue tip and then detached cells were removed by excluding media and washing with phosphate-buffered saline. Media were replaced to DMEM supplemented with $2 \%$ FBS and cells were irradiated with a proton beam in a dose of $2,4,8$ or $16 \mathrm{~Gy}$. The wound areas were observed and captured at 0 and $24 \mathrm{~h}$ after proton beam irradiation, respectively.

Animal model and tumorigenesis. Animal experiments were performed with the approval of the Institutional Animal Care and Use Committee at Dongguk University (IACUC2014-008). Female BALB/c mice were purchased from Orient Bio Inc. (Gapyung, Korea) and housed under a $12 \mathrm{~h}$ light/dark cycle at $25 \pm 2^{\circ} \mathrm{C}$ with a relative humidity of $50 \pm 5 \%$. After adaptation of one week, $1 \times 10^{5} 4 \mathrm{~T} 1$ murine breast cancer cells were implanted into the mammary fat pad of 5-week-old female BALB/c mice to establish the orthotopic animal breast cancer model. When the tumor volumes reached $\sim 200 \mathrm{~mm}^{3}$, mice were randomly divided into 4 groups: 0 (control), 10 , 20 and 30 Gy. Mice were protected by an acryl plate $(50 \mathrm{~mm}$ thickness) and mammary tumors were selectively irradiated with a proton beam at a dose of 10,20 or 30 Gy using the $100 \mathrm{MeV}$ proton accelerator. Tumor sizes and body weights were measured twice a week. Tumor volume $\left(\mathrm{mm}^{3}\right)$ was calculated with the following formula: tumor volume $=$ width $^{2}$ $\mathrm{x}$ length $\mathrm{x} 0.5$. Mice were sacrificed after 14 days and breast tumors and lungs were dissected.

Quantitative RT-PCR. The proton beam irradiation-mediated changes in metastasis-regulating genes, such as MMP-9, MMP-2, UPA, uPAR, cyclooxygenase (COX)-2, and VEGF, were analyzed. Total RNA was extracted from breast tumor tissues using the easy-BLUE ${ }^{\mathrm{TM}}$ Total RNA Extraction kit (iNtRON Biotechnology Inc., Sungnam, Korea) according to the manufacturer's protocol. cDNA was synthesized with Goscript $^{\mathrm{TM}}$ Reverse Transcriptase (Promega, Madison, WI, USA). Real-time PCR reactions were performed with QGreen 2X SybrGreen Master Mix (Cellsafe, Suwon, Korea) using $\mathrm{Eco}^{\mathrm{TM}}$ Real-Time PCR (Illumina, San Diego, CA, USA). The reaction was preheated at $95^{\circ} \mathrm{C}$ for $10 \mathrm{~min}$, followed by 40 cycles of $90^{\circ} \mathrm{C}$ for $10 \mathrm{sec}, 60^{\circ} \mathrm{C}$ for $15 \mathrm{sec}$, and $72^{\circ} \mathrm{C}$ for $20 \mathrm{sec}$; products were verified by melting curve analysis. Relative expression levels compared with control were automatically evaluated by Eco Software v3.1.7. Glyceraldehyde 3-phosphate dehydrogenase (GAPDH) was used as an internal control. Sequences of the primers used for the reaction were as follows: MMP-9: forward, 5'-GCGAGTG TCTCCCTCAAACG-3'; reverse, 5'-TCCTCACGGTGATGCT GTTC-3'. MMP-2: forward, 5'-GCTGATACTGACACTGGT ACTG-3'; reverse, 5'-CAATCTTTT CTGGGAGCTC-3'. uPA: forward, 5'-ATGCATGGTGCATGACTGCTCT-3'; reverse, 5'-ATTCGTGCAAGATGAGCTGCTC-3'. uPAR: forward, 5'-CAGAGCACAGAAAGGAGCTTGA-3'; reverse, 5'-CTG AAAGGTCTGGTTGCTATGGA-3'. COX-2: forward, 5'-CCT GCTGCCCGACACCTTCA-3'; reverse, 5'-AGCAACCCGGC CAGCAATCT-3'. VEGF: forward, 5'-GTACCTCCACCAT GCCAAGT-3'; reverse, 5'-GCATTCACATCTGCTGTGCT-3'. 

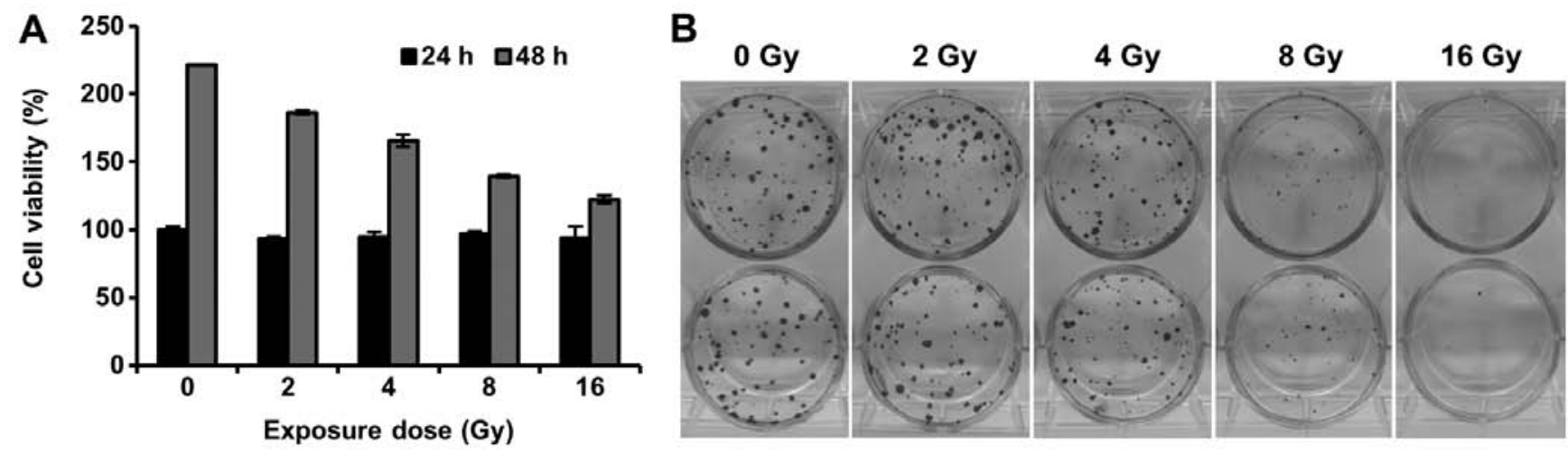

Figure 1. A proton beam decreased proliferation and clonogenic survival of 4T1 murine breast cancer cells. (A) Cell viability of 4T1 cells after irradiation with a proton beam. Cells were seeded in 96 -well plates and irradiated with 2, 4, 8 or 16 Gy proton beam and then cell viability was measured 24 and $48 \mathrm{~h}$ later with MTT reagent, respectively. (B) Representative images of colonies. Cells were seeded and grown for $24 \mathrm{~h}$ prior to irradiation by $2,4,8$ or $16 \mathrm{~Gy}$ proton beam, respectively. Cells were additionally grown for 6 days, and then the formed colonies were fixed with $10 \%$ formalin and stained with $1 \%$ crystal blue.
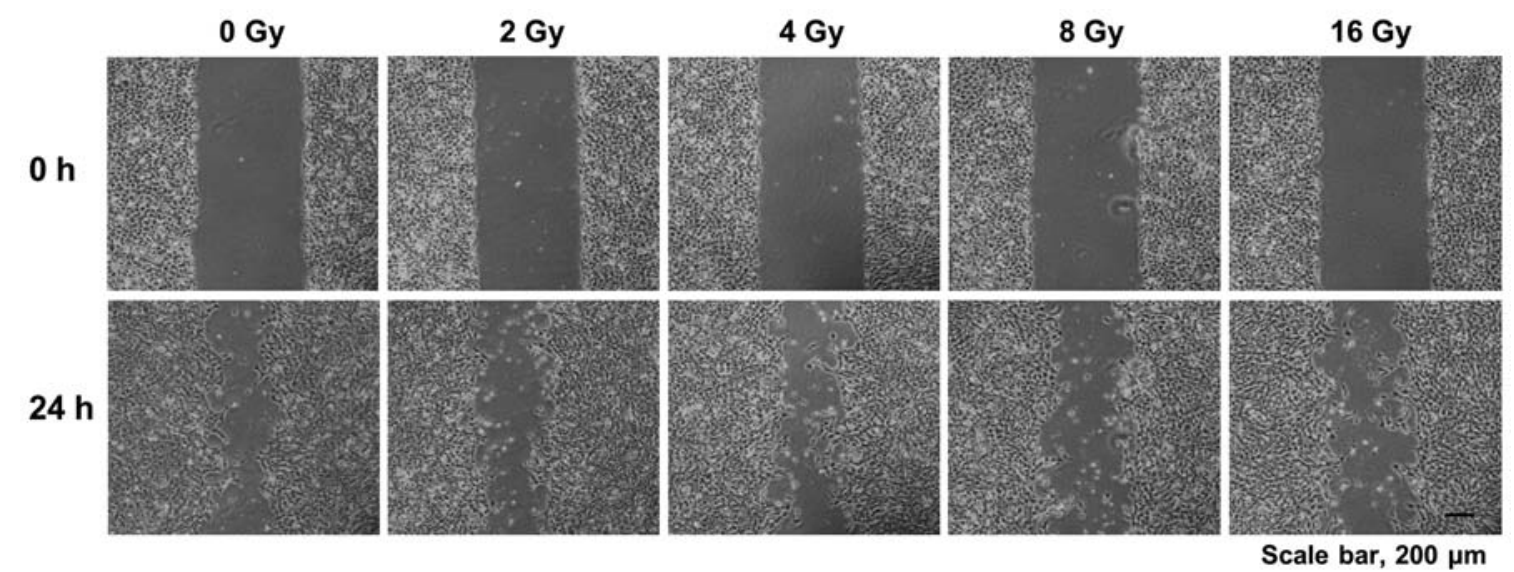

Figure 2. A proton beam inhibited the cell migration of $4 \mathrm{~T} 1$ murine breast cancer cells. Cells were plated in 6-well plates and irradiated with $2,4,8$ or $16 \mathrm{~Gy}$ proton beam. Wounded areas were captured at 0 and $24 \mathrm{~h}$ after proton beam irradiation using an optical microscope.

GAPDH: forward, 5'-GTATGACTCCACTCACGGCAAA-3'; reverse, 5'-GGTCTCGCTCCTGGAAGATG-3'.

Pathological analysis. To observe metastasized tumor cells in the lung, lung tissues were collected from mice, fixed with $10 \%$ formalin, and embedded in paraffin. Then the paraffinembedded lung tissues were cut into 4-mm-thick slices and stained with haematoxylin and eosin (H\&E). Tissue sections were randomly selected and tumor nodules were counted using optical microscopes; the sizes of the tumor nodules were also measured. The sizes of the tumor nodules were calculated with the following formula: size of tumor nodule $=$ (the longest diameter + the shortest diameter)/2.

Statistical analysis. Statistical significance was determined using the Student's t-test or one-way ANOVA. All cell experiments were conducted in triplicate, and results are presented as mean $\pm \mathrm{SD}$. P-values of $<0.05$ were considered significant.

\section{Results}

Effects on proliferation and survival of $4 T 1$ murine breast cancer cells. We assessed the effect of a proton beam on cell prolifera- tion and clonogenic survival in 4T1 murine breast cancer cells. Although cell proliferation was not affected by proton beam irradiation within $24 \mathrm{~h}$, the proton beam inhibited the proliferation at $48 \mathrm{~h}$ in MTT assay (Fig. 1A). In addition, the colony forming assay showed that clonogenic viability was significantly decreased by proton beam irradiation (Fig. 1B). These results are consistent with several studies that revealed inhibition of cell growth by proton beam irradiation in human solid tumors, such as pancreas, prostate, and breast cancer $(14,15,20)$.

Effects on cell migration in $4 T 1$ murine breast cancer cells. Cell migration is crucial for cancer cells to metastasize from a primary tumor tissue to distant sites in the body. Therefore, we also evaluated the effects of proton beam irradiation on cell migration of $4 \mathrm{~T} 1$ murine breast cancer cells in the wound healing assay. We found that cell migration of 4T1 murine breast cancer cells was effectively prevented by proton beam irradiation ranging from 2 to $16 \mathrm{~Gy}$ (Fig. 2). This result demonstrates that a proton beam may suppress the metastatic potential of $4 \mathrm{~T} 1$ murine breast cancer cells.

Effects on breast tumor growth and lung metastasis in $4 T 1$ orthotopic animal model. In vitro experiments showed the 

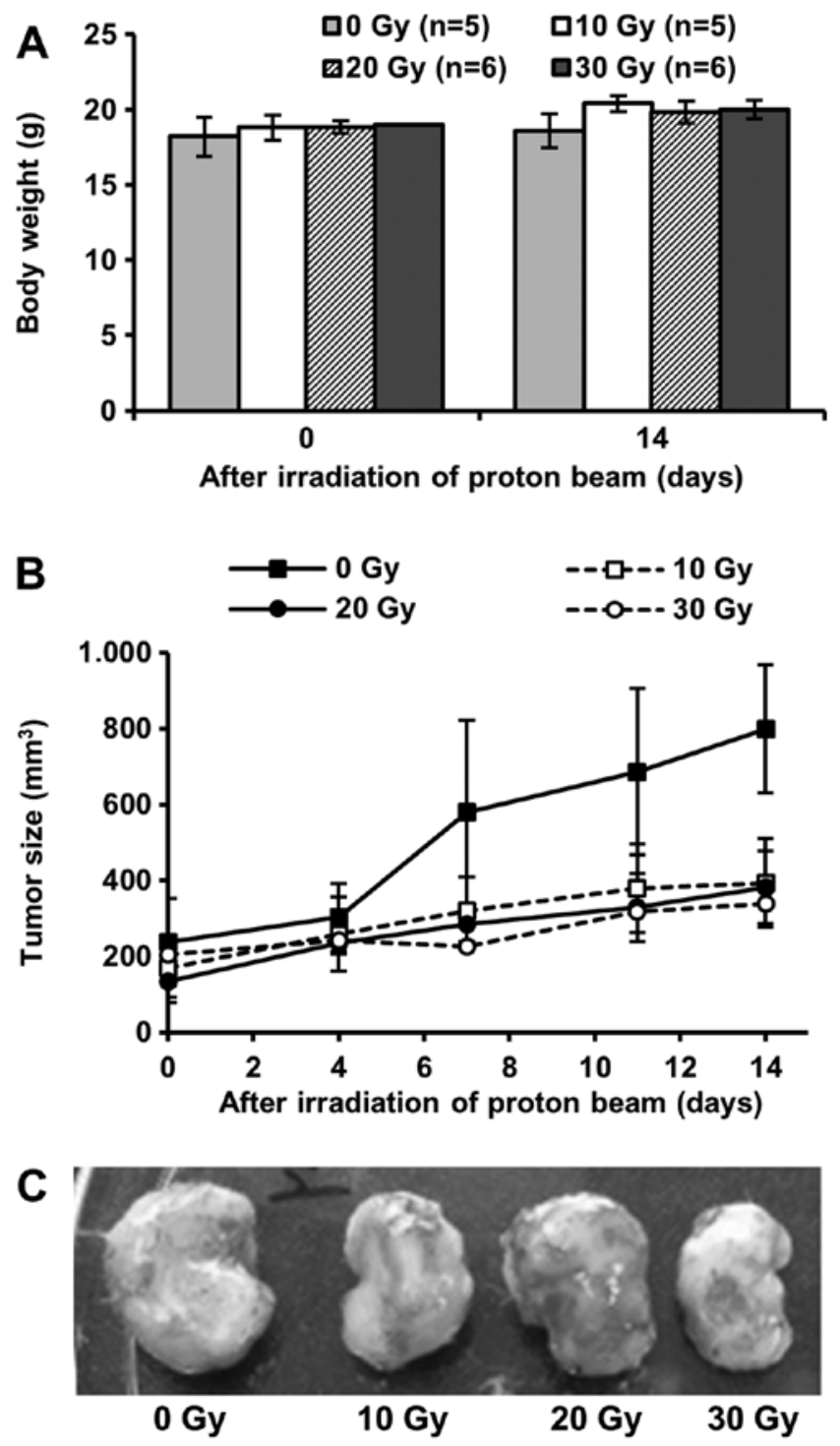

Figure 3. Proton beam delayed tumor growth in 4T1 orthotopic breast cancer model. (A) Changes of mouse body weight. Values indicate average body weight in each group. (B) Changes of breast tumor growth by proton beam irradiation. Tumor sizes were measured every 3 or 4 days. (C) Image of the breast tumor in each group. Breast tumor in each group was irradiated with indicated proton beam dose and then additionally grown for 14 days.

suppressive effects of proton beam irradiation against cell proliferation, clonogenic survival and cell migration in 4T1 murine breast cancer cells (Figs. 1 and 2). Based on the results, we assessed the effects of proton beam irradiation on breast tumor growth and lung metastasis in BALB/c mice implanted with 4T1 murine breast cancer cells. The growth of breast tumors irradiated by a proton beam was significantly repressed without body weight changes (Fig. 3A and B). Furthermore, fewer tumor nodules were found in lungs of mice irradiated with 30 Gy proton beam (Fig. 4A). Also, although not significant, the size of tumor nodules in mice irradiated with 30 Gy proton beam was smaller than others (Fig. 4B and C). However, 10 and 20 Gy proton beam could not prevent lung metastasis of breast cancer. The results correspond with those of in vitro cell proliferation and cell migration assays (Figs. 1 and 2).
Effects on metastatic gene expression in 4T1-implanted breast tumors. We determined the proton beam irradiation-mediated change in transcription of metastatic genes, such as COX-2, uPA, uPAR, MMP-9 and MMP-2, in breast tumors. As shown in Fig. 5A and B, COX-2, UPA and UPAR expressions were inhibited by $30 \mathrm{~Gy}$ proton beam irradiation. However, proton beams did not suppress MMP-2 and -9 transcription (Fig. 5C). Surprisingly, mRNA expression of COX-2, uPA, MMP-2 and MMP-9 genes were enhanced in breast tumor irradiated with 20 Gy proton beam (Fig. 5A-C), suggesting that optimized dose irradiation of a proton beam is important for improving cancer treatment and preventing metastasis. We also clarified the effect of proton beam irradiation on VEGF gene expression. Lower levels of VEGF mRNA were also observed in breast tumors irradiated with 30 Gy proton beam (Fig. 5D). The suppression of VEGF mRNA expression corresponded with the change of metastatic gene expression by proton beam irradiation.

\section{Discussion}

Radiation therapy is a treatment technique to kill cancer cells and prevent tumor growth using high energy beams, such as $\mathrm{X}$-ray, gamma ray or proton beam. X-ray is the most common energy source for radiation therapy. However, this technique can induce various side effects, such as radionecrosis, blood dyscrasia, inappropriate hormone secretion and so on, as a result of normal tissues damage caused by the physical characteristics of X-ray. Proton beam therapy is a new high technology radiation therapy. A proton beam enters the body, releasing the highest energy when it reaches the target and then the energy quickly fades away. Because of these physical properties, proton beam irradiation is used to treat various cancers, including breast, bladder, head and neck, to minimize normal tissue damage. Global proton beam facilities to treat cancer are increasing (21).

We found in previous studies that metastatic potential in MCF-7 and MDA-MB-231 human breast cancer cells was diminished by proton beam irradiation and cell viabilities in both were also decreased $(12,16,17,22)$. The results demonstrated that a proton beam should increase the efficacy of breast cancer treatment while inhibiting metastasis. However, previous studies did not elucidate the in vivo anti-metastatic potential of a proton beam. Here, we assessed the effects of proton beam irradiation on in vivo breast cancer metastasis by using the 4T1 orthotopic breast cancer model. 4T1 cells are known as an adequate cancer cell model to research human breast cancer because the cells can easily develop into a primary tumor in the mammary gland and spontaneously metastasize with a similar pattern as human breast cancer metastasis (23). Therefore, we used 4T1 cells to clarify the anti-metastatic effects of a proton beam. Prior to in vivo analysis, we found that proton beam irradiation prevented cell proliferation and clonogenic survival in an in vitro system with 4T1 murine breast cancer cells (Fig. 1). Also, cell migration of 4T1 murine breast cancer cells was suppressed by proton beam irradiation in the wound healing assay (Fig. 2). The results show that metastatic activity in 4T1 murine breast cancer cells should be prevented by proton beam irradiation. Therefore, the data support that 4T1 murine breast cancer cells are suitable 

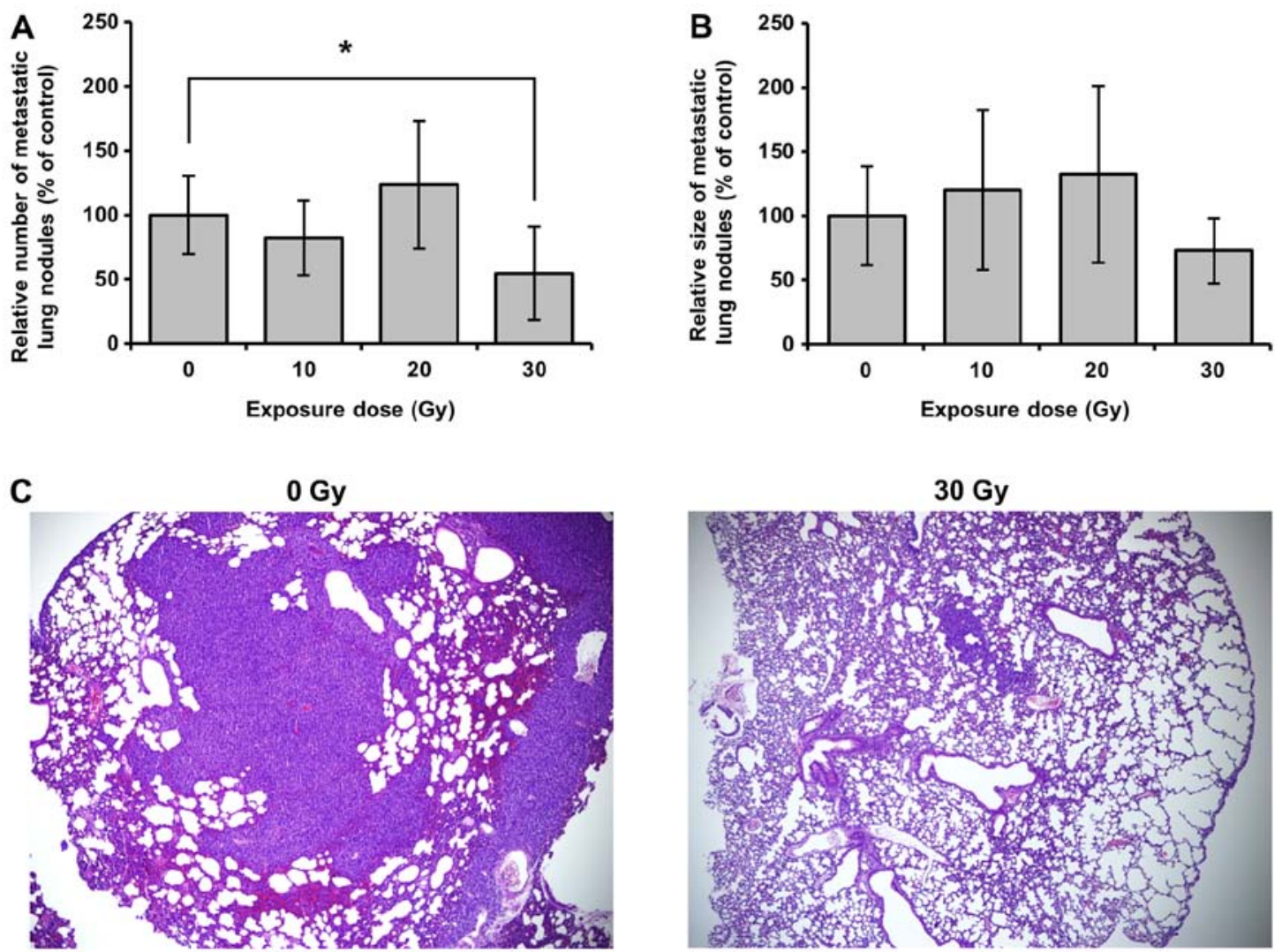

Figure 4. Lung metastasis was significantly suppressed by $30 \mathrm{~Gy}$ proton beam. The tumor nodules were microscopically observed. (A) Relative number of metastasized tumor nodules. The values are presented as relative percent compared to control ( $0 \mathrm{~Gy})$. (B) Relative size of metastasized tumor nodules. The values are presented as relative percent against control ( 0 Gy). Significant difference was considered as P-values $<0.05$. ${ }^{*} \mathrm{P}<0.05$. (C) Lung tissues in $4 \mathrm{~T} 1$ orthotopic breast cancer model were observed by optical microscope. Tissues were stained with haematoxylin and eosin and pathologically analyzed in a blinded manner. $0 \mathrm{~Gy}$ indicates lung tissue in control mouse and $30 \mathrm{~Gy}$ indicates lung tissue in mouse irradiated with $30 \mathrm{~Gy}$ proton beam. Magnification, $\mathrm{x} 40$.
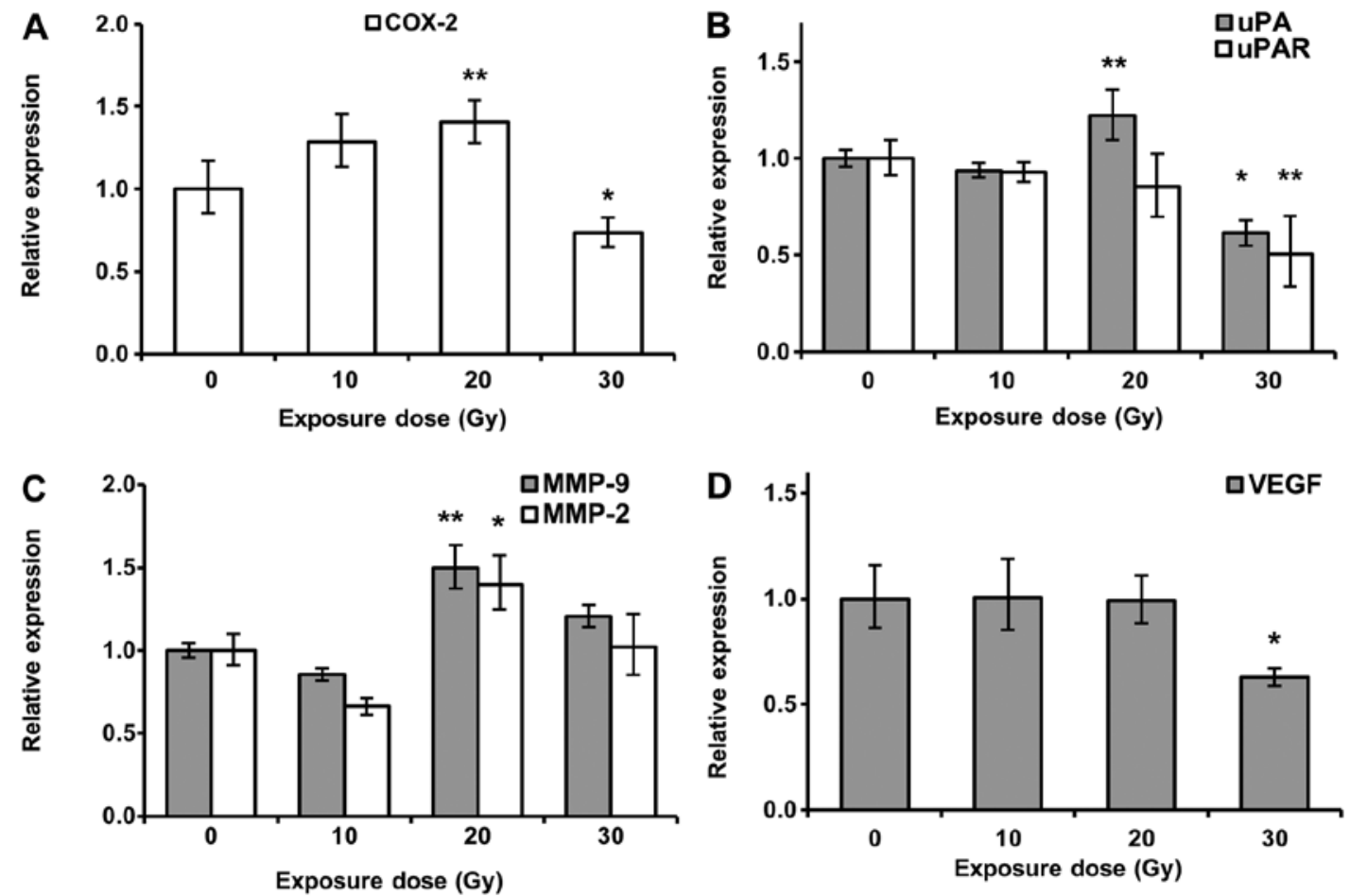

Figure 5. Metastasis-enhancing gene transcription was significantly repressed by $30 \mathrm{~Gy}$ proton beam in $4 \mathrm{~T} 1$ orthotopic breast tumor. Relative expression levels of metastasis-enhancing genes and VEGF gene were determined by quantitative real-time PCR. Relative expression level of (A) COX-2, (B) uPA and uPAR, (C) MMP-9 and MMP-2 and (D) VEGF in 4T1 orthotopic breast tumors in BALB/c mice irradiated with 10, 20, or 30 Gy proton beam, respectively. Values are presented as means \pm SD. ${ }^{*}$ and ${ }^{* *}$ indicate $\mathrm{P}$-value $<0.05$ and $\mathrm{P}$-value $<0.01$, respectively. 
to investigate the in vivo anti-metastatic effects of a proton beam. Based on these results, we established the BLAB/c 4T1 orthotopic breast cancer model and then irradiated the breast cancers with 10, 20 and 30 Gy proton beams.

The 5-year survival rate in breast cancer patients is predominantly determined by whether or not the cancer metastasizes (24). Therefore, inhibition of metastatic potential in breast cancer is important to improve prognosis of patients. Wang et al in 2012 showed that endothelial-mesenchymal transition (EMT) in human epithelial cells was enhanced by proton beam irradiation of $<2 \mathrm{~Gy}$ (25). Although not all cancer cells undergo EMT prior to metastasis, many cancers go through EMT for successful metastasis (26-28). Therefore, the data demonstrate that $<2$ Gy proton beam should enhance metastatic potential in cancer. Here, we found that breast tumor growth was significantly suppressed by proton beam irradiation without changes of body weight (Fig. 3), and 30 Gy proton beam significantly inhibited lung metastasis in the animal model (Fig. 4A and C). However, although 10 and 20 Gy proton beams critically delayed breast tumor growth, neither dose of proton beams prevented lung metastasis of breast cancer in the animal model (Figs. 3 and 4A and C). Consequently, both studies implicate the importance of appropriate exposure dose to improve therapeutic efficacy in proton beam therapy.

We further investigated the effects of proton beam irradiation on the expression of metastasis-regulating genes, such as COX-2, uPA and UPAR, in 4T1 orthotopic breast cancer model. The results showed lower levels of COX-2, uPA and UPAR transcription in breast cancer in mice irradiated with $30 \mathrm{~Gy}$ proton beam (Fig. 5A and B). Furthermore, 30 Gy proton beam downregulated VEGF mRNA expression (Fig. 5D). However, the expression of MMP-2 and -9 genes was not changed (Fig. 5C). The importance of these factors in metastasis has been well explained in previous studies. Enhanced COX-2 expression triggers metastasis with the induction of VEGF expression, and anti-metastatic agents inhibit COX-2 and MMPs expression (29-31). Other reports demonstrated that the uPA system facilitates cell invasion, adhesion and metastasis in breast cancer $(32,33)$. Furthermore, Andres et al suggested that the uPA system should be a useful biomarker for predicting the prognosis of breast cancer patients (34). Based on these reports, metastatic potential is closely correlated with the expression level of these factors and should be suppressed by their downregulation. Therefore, the present results demonstrate that $30 \mathrm{~Gy}$ proton beam prevents lung metastasis of breast cancer through the suppression of COX-2, uPA, uPAR and VEGF in the 4T1 orthotopic breast cancer animal model.

In conclusion, we found that cell proliferation, viability and migration of 4T1 breast cancer cells were significantly decreased by proton beam irradiation. Also, in vivo research showed that tumor growths in 4T1 orthotopic breast cancer model were drastically delayed by proton beam irradiation. Furthermore, $30 \mathrm{~Gy}$ proton beam inhibited lung metastasis via suppression of COX-2, uPA, uPAR and VEGF gene expression. However, prevention of lung metastasis was not observed in mice irradiated with 10 and 20 Gy proton beams. Taken together, our data suggest that a proton beam is a useful tool in metastatic breast cancer treatment and demonstrate that selection of a suitable exposure dose is important to improve therapeutic efficacy through the inhibition of metastasis to distant organs in breast cancer.

\section{Acknowledgements}

This study was supported by the National Research Foundation of Korea (NRF) grant funded by the Ministry of Science, ICT and future Planning (2014M2B2A4030083).

\section{References}

1. Weigelt B, Peterse JL and van't Veer LJ: Breast cancer metastasis: Markers and models. Nat Rev Cancer 5: 591-602, 2005.

2. Howlader NNA, Krapcho M, Garshell J, Miller D, Altekruse SF, Kosary CL, Yu M, Ruhl J, Tatalovich Z, Mariotto A, et al (eds): SEER Cancer Statistics Review, 1975-2013. http://seer.cancer. gov/csr/1975 2013. Accessed April 15, 2016.

3. Westermarck J and Kahari VM: Regulation of matrix metalloproteinase expression in tumor invasion. FASEB J 13: 781-792, 1999.

4. Lee KS, Shin JS and Nam KS: Starfish polysaccharides downregulate metastatic activity through the MAPK signaling pathway in MCF-7 human breast cancer cells. Mol Biol Rep 40: 5959-5966, 2013.

5. Yang F, Hu M, Lei Q, Xia Y, Zhu Y, Song X, Li Y, Jie H, Liu C, Xiong Y, et al: Nifuroxazide induces apoptosis and impairs pulmonary metastasis in breast cancer model. Cell Death Dis 6: e1701, 2015.

6. Li X, Kong X, Wang Y and Yang Q: BRCC2 inhibits breast cancer cell growth and metastasis in vitro and in vivo via downregulating AKT pathway. Cell Death Dis 4: e757, 2013.

7. Daniele A, Zito AF, Giannelli G, Divella R, Asselti M, Mazzocca A, Paradiso A and Quaranta M: Expression of metalloproteinases MMP-2 and MMP-9 in sentinel lymph node and serum of patients with metastatic and non-metastatic breast cancer. Anticancer Res 30: 3521-3527, 2010.

8. Harbeck N, Schmitt M, Kates RE, Kiechle M, Zemzoum I, Jänicke $\mathrm{F}$ and Thomssen $\mathrm{C}$ : Clinical utility of urokinase-type plasminogen activator and plasminogen activator inhibitor-1 determination in primary breast cancer tissue for individualized therapy concepts. Clin Breast Cancer 3: 196-200, 2002.

9. Huang S, New L, Pan Z, Han J and Nemerow GR: Urokinase plasminogen activator/urokinase- specific surface receptor expression and matrix invasion by breast cancer cells requires constitutive $\mathrm{p} 38$ alpha mitogen-activated protein kinase activity. J Biol Chem 275: 12266-12272, 2000.

10. Janicke F, Prechtl A, Thomssen C, Harbeck N, Meisner C, Untch M, Sweep CGJF, Selbmann H-K, Graeff H and Schmitt M: Randomized adjuvant chemotherapy trial in high-risk, lymph node-negative breast cancer patients identified by urokinasetype plasminogen activator and plasminogen activator inhibitor type 1. J Natl Cancer Inst 93: 913-920, 2001.

11. Look MP: Pooled analysis of UPA and PAI-1 for prognosis in primary breast cancer patients. EORTC Receptor and Biomarker Study Group. Int J Biol Markers 15: 70-72, 2000.

12. Lee KS, Lee DH, Chun SY and Nam KS: Metastatic potential in MDA-MB-231 human breast cancer cells is inhibited by proton beam irradiation via the Akt/nuclear factor-kappaB signaling pathway. Mol Med Rep 10: 1007-1012, 2014.

13. Foote RL, Stafford SL, Petersen IA, Pulido JS, Clarke MJ, Schild SE, Garces YI, Olivier KR, Miller RC, Haddock MG, et al: The clinical case for proton beam therapy. Radiat Oncol 7: 174, 2012.

14. Alan Mitteer R, Wang Y, Shah J, Gordon S, Fager M, Butter P-P, Jun Kim H, Guardiola-Salmeron C, Carabe-Fernandez A and Fan Y: Proton beam radiation induces DNA damage and cell apoptosis in glioma stem cells through reactive oxygen species. Sci Rep 5: 13961, 2015.

15. Narang H, Kumar A, Bhat N, Pandey BN and Ghosh A: Effect of proton and gamma irradiation on human lung carcinoma cells: Gene expression, cell cycle, cell death, epithelial-mesenchymal transition and cancer-stem cell trait as biological end points. Mutat Res 780: 35-46, 2015.

16. Lee KS, Shin JS and Nam KS: Effect of proton beam irradiation on the regulation of metastasis-enhancing factors in MCF-7 human breast cancer cells. J Korean Phys Soc 63: 1373-1378, 2013. 
17. Lee KS, Shin JS, Shon YH and Nam KS: Anti-angiogenic activity in metastasis of human breast cancer cell irradaited by a proton beam. J Korean Phys Soc 61: 268-272, 2012.

18. Miller FR: Tumor subpopulation interactions in metastasis. Invasion Metastasis 3: 234-242, 1983.

19. Miller FR, Miller BE and Heppner GH: Characterization of metastatic heterogeneity among subpopulations of a single mouse mammary tumor: Heterogeneity in phenotypic stability. Invasion Metastasis 3: 22-31, 1983.

20. Lee KB, Lee JS, Park JW, Huh TL and Lee YM: Low energy proton beam induces tumor cell apoptosis through reactive oxygen species and activation of caspases. Exp Mol Med 40: 118-129, 2008.

21. Jermann M: Particle therapy statistics in 2014. Int J Particle Ther 2: $50-54,2015$

22. Lee KS, Mo JY, Shon YH and Nam KS: Inhibition of metastatic activities in human breast cancer cells irradiated by a proton beam. J Korean Phys Soc 59: 653-656, 2011.

23. Pulaski BA and Ostrand-Rosenberg S: Mouse 4T1 breast tumor model. Curr Protoc Immunol Chapter 20: Unit 20 22, 2001. doi: 10.1002/0471142735.im2002s39.

24. Nieves-Alicea R, Colburn NH, Simeone AM and Tari AM: Programmed cell death 4 inhibits breast cancer cell invasion by increasing tissue inhibitor of metalloproteinases-2 expression. Breast Cancer Res Treat 114: 203-209, 2009.

25. Wang M, Hada M, Saha J, Sridharan DM, Pluth JM and Cucinotta FA: Protons sensitize epithelial cells to mesenchymal transition. PLoS One 7: e41249, 2012.

26. Heerboth S, Housman G, Leary M, Longacre M, Byler S, Lapinska K, Willbanks A and Sarkar S: EMT and tumor metastasis. Clin Transl Med 4: 6, 2015.

27. Wallerand H, Cai Y, Wainberg ZA, Garraway I, Lascombe I, Nicolle G, Thiery J-P, Bittard H, Radvanyi F and Reiter RR: Phospho-Akt pathway activation and inhibition depends on $\mathrm{N}$-cadherin or phospho-EGFR expression in invasive human bladder cancer cell lines. Urol Oncol 28: 180-188, 2010.
28. Bailey JM, Singh PK and Hollingsworth MA: Cancer metastasis facilitated by developmental pathways: Sonic hedgehog, Notch, and bone morphogenic proteins. J Cell Biochem 102: 829-839, 2007.

29. Kundu N and Fulton AM: Selective cyclooxygenase (COX)-1 or COX-2 inhibitors control metastatic disease in a murine model of breast cancer. Cancer Res 62: 2343-2346, 2002.

30. Morita Y, Hata K, Nakanishi M, Nishisho T, Yura Y and Yoneda T: Cyclooxygenase-2 promotes tumor lymphangiogenesis and lymph node metastasis in oral squamous cell carcinoma. Int J Oncol 41: 885-892, 2012.

31. Kang JH, Song KH, Jeong KC, Kim S, Choi C, Lee C and Oh S: Involvement of Cox-2 in the metastatic potential of chemotherapy-resistant breast cancer cells. BMC Cancer 11: 334, 2011.

32. Stillfried GE, Saunders DN and Ranson M: Plasminogen binding and activation at the breast cancer cell surface: The integral role of urokinase activity. Breast Cancer Res 9: R14, 2007.

33. De Cremoux P, Grandin L, Dieras V, Savignoni A, Degeorges A, Salmon R, Bollet MA, Reyal F, Sigal-Zafrani B, VincentSalomon A, et al: Urokinase-type plasminogen activator and plasminogen-activator-inhibitor type 1 predict metastases in good prognosis breast cancer patients. Anticancer Res 29: 1475-1482, 2009.

34. Andres SA, Edwards AB and Wittliff JL: Expression of urokinase-type plasminogen activator (uPA), its receptor (uPAR), and inhibitor (PAI-1) in human breast carcinomas and their clinical relevance. J Clin Lab Anal 26: 93-103, 2012. 